\title{
Association of Primary Biliary Cirrhosis-autoimmune Hepatitis Overlap Syndrome with Immune Thrombocytopenia and Graves' Disease
}

\author{
Ryosuke Koyamada ${ }^{1}$, Takakazu Higuchi ${ }^{1}$, Ayako Kitada ${ }^{2}$, Tomoko Nakagawa ${ }^{3}$, \\ Takashi Ikeya ${ }^{4}$, Sadamu Okada ${ }^{1}$ and Yoshiyuki Fujita ${ }^{4}$
}

\begin{abstract}
A 54-year-old woman suffering from pruritus for five years was diagnosed to have Graves' disease and immune thrombocytopenia (ITP) associated with primary biliary cirrhosis (PBC)-autoimmune hepatitis (AIH) overlap syndrome, which was confirmed histologically after a prompt recovery in the platelet count number following steroid therapy. The association between PBC-AIH overlap syndrome and ITP has been rarely reported and the additional association with Graves' disease has not yet been reported. An underlying global derangement of autoimmunity or shared genetic susceptibility was suspected.
\end{abstract}

Key words: primary biliary cirrhosis, autoimmune hepatitis, PBC-AIH overlap syndrome, immune thrombocytopenia, Graves' disease

(Intern Med 54: 2013-2016, 2015)

(DOI: 10.2169/internalmedicine.54.4405)

\section{Introduction}

Thrombocytopenia is a common hematological complication of chronic liver diseases $(1,2)$. Multiple factors have been proposed to contribute to the development of thrombocytopenia, including splenic sequestration of platelets, bone marrow suppression, and a decreased activity of thrombopoietin $(1,2)$. Primary biliary cirrhosis (PBC) and autoimmune hepatitis (AIH) are two types of chronic autoimmune liver diseases. PBC is characterized by progressive inflammatory damage to the biliary epithelial cells, elevated biliary tract enzymes and positive anti-mitochondrial antibody (AMA). PBC is known to frequently have other autoimmune phenomena $(3,4)$. Anti-platelet autoantibodies are detected in approximately $40 \%$ of patients and it is assumed that the immune-mediated destruction of platelets contributes to the thrombocytopenia in a considerable portion of patients with PBC $(5,6)$. Conversely, AIH is an autoimmune chronic active hepatitis with elevated transaminases, serum immunoglobulin (Ig) G levels, positive anti-nuclear antibody (ANA) and smooth muscle antibody (SMA) (7). AIH is also known to be frequently associated with additional autoimmune phenomena (8); however, unlike PBC, the frequency of antiplatelet antibodies among patients with $\mathrm{AIH}$ has not been reported. PBC-AIH overlap syndrome is a simultaneous or consecutive association of $\mathrm{PBC}$ and $\mathrm{AIH}(9,10)$ and, like $\mathrm{PBC}$ and $\mathrm{AIH}$, is frequently associated with extrahepatic autoimmune disorders (11); however, the frequency of antiplatelet antibodies has not yet been studied despite occasional case reports of the association with immune thrombocytopenia (ITP) (12-15).

We herein report a case of PBC-AIH overlap syndrome associated with ITP and Graves' disease. We found one case of PBC-AIH overlap syndrome and ITP associated with Hashimoto's disease in the literature (12); however, there has been no previous reported case associated with Graves' disease.

\section{Case Report}

A 54-year-old Japanese woman was admitted to our hos-

${ }^{1}$ Division of Hematology, St. Luke's International Hospital, Japan, ${ }^{2}$ Internal Medicine, St. Luke's International Hospital, Japan, ${ }^{3}$ Division of Endocrinology, St. Luke's International Hospital, Japan and ${ }^{4}$ Division of Gastroenterology, St. Luke's International Hospital, Japan

Received for publication October 28, 2014; Accepted for publication January 15, 2015

Correspondence to Dr. Takakazu Higuchi, takhig@luke.ac.jp 


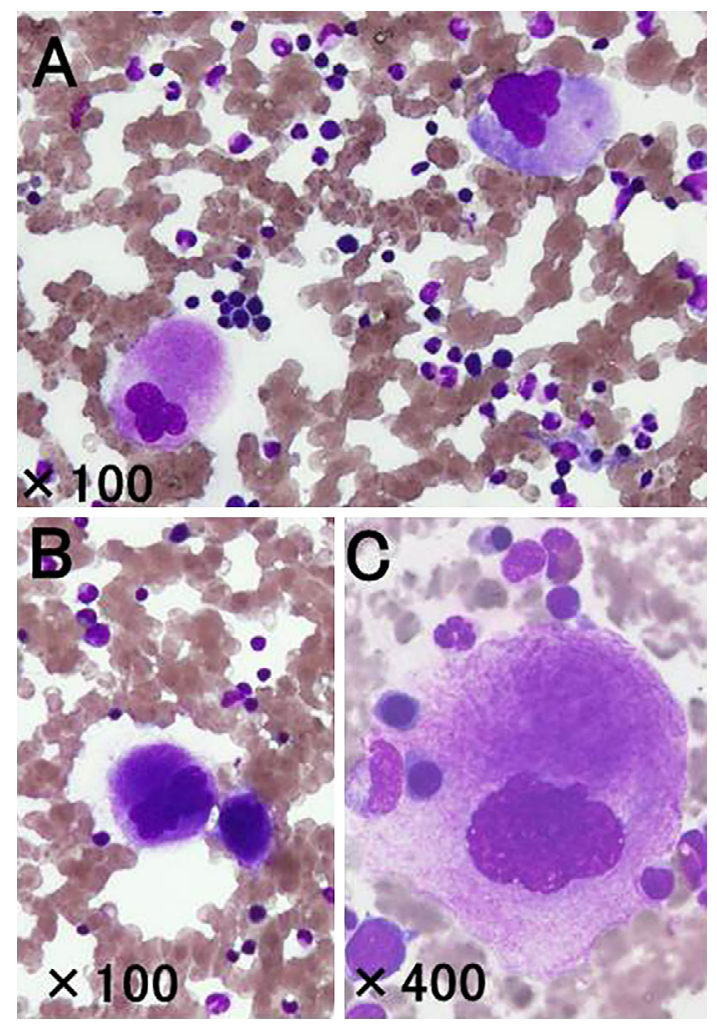

Figure 1. Bone marrow films on admission. The bone marrow was normocellular with normal erythroid and myeloid cells $(A, 100 \times)$. The megakaryocytes were increased in number and both small ones with hypolobulated nuclei and scanty cytoplasms $(B, 100 \times)$ and larger ones with ample cytoplasms $(C$, $400 \times$ ) were observed. Few platelets were visualized around and near the megakaryocytes (Wright-Giemsa stain).

pital for the evaluation of thrombocytopenia. She had been suffering from pruritus for five years and noticed palpitation and hair loss six months previously. She had also lost $20 \%$ of her body weight in six months. Two weeks before admission, she visited another hospital and the laboratory tests revealed that the thyroid stimulating hormone level was below $0.01 \mu \mathrm{IU} / \mathrm{mL}$, free thyroxin $7.4 \mathrm{ng} / \mathrm{dL}$, free triiodothyronine $14.8 \mathrm{pg} / \mathrm{mL}$, and anti-thyrotropin receptor antibody $6.6 \mathrm{IU} /$ L, and she was diagnosed with Graves' disease. The blood test also revealed moderate thrombocytopenia with a platelet count of $67 \times 10^{9} / \mathrm{L}$ and liver dysfunction with a total bilirubin level $2.1 \mathrm{mg} / \mathrm{dL}$, aspartate aminotransferase (AST) $73 \mathrm{U} /$ $\mathrm{L}$, alanine aminotransferase (ALT) $61 \mathrm{U} / \mathrm{L}$, alkaline phosphatase (ALP) 1,030 U/L, and $\gamma$-glutamyl transferase $(\gamma$ GTP) 162 U/L. Propranolol and thiamazole treatment was initiated. One day before admission, the follow-up blood test revealed that her platelet count had further decreased to $21 \times$ $10^{9} / \mathrm{L}$ and she was referred and admitted to our hospital.

On admission, fine tremor of the fingers was noted. The thyroid gland was diffusely enlarged, and the liver and the spleen were palpable below the lower costal margins. Neither subcutaneous nor mucosal hemorrhage was seen. The laboratory data showed that the patient's platelet count was $20 \times 10^{9} / \mathrm{L}$, red blood cell count $4.5 \times 10^{12} / \mathrm{L}$, hemoglobin level
$12.9 \mathrm{~g} / \mathrm{dL}$, hematocrit $38.7 \%$, and white blood cell count $6.4 \times 10^{9} / \mathrm{L}$ with normal differentials. The coagulation tests were normal. The total bilirubin level was $2.4 \mathrm{mg} / \mathrm{dL}$, AST $63 \mathrm{U} / \mathrm{L}$, ALT $73 \mathrm{U} / \mathrm{L}$, ALP 1,163 U/L (ALP1 6\%, ALP2 $53 \%$, ALP3 37\%, ALP5 4\%), lactate dehydrogenase $235 \mathrm{U} /$ $\mathrm{L}$, and $\gamma$-GTP $162 \mathrm{U} / \mathrm{L}$. The serum IgG level was $1,709 \mathrm{mg} /$ $\mathrm{dL}, \mathrm{IgM} 207 \mathrm{mg} / \mathrm{dL}$, and ANA was positive with a ratio of 1:640 with speckled, homogenous, and granular patterns. AMA was positive at $40 \mathrm{mg} / \mathrm{dL}$, and platelet-associated (PA) IgG was $63.3 \mathrm{ng} / 10^{7}$ cells (reference range: 5.0-25.0). Hepatitis $\mathrm{B}$ surface antigen and hepatitis $\mathrm{C}$ virus antibody were negative. Both anti-Helicobacter pylori $\mathrm{IgG}$ and the urea breath test were negative. Abdominal ultrasonography and computed tomography revealed mild splenomegaly. Bone marrow aspiration showed a normocellular marrow with normal differentiation of both the erythroid and myeloid series without dysplasia and an increased number of megakaryocytes (Fig. 1). These findings were compatible with ITP. In addition, based on the abnormal liver function tests, positive AMA and ANA, an elevated IgG concentration, and negative hepatitis virus markers, PBC-AIH overlap syndrome was considered to be complicated; however, thrombocytopenia discouraged us from performing a liver biopsy at that time. Prednisolone $(1 \mathrm{mg} / \mathrm{kg})$ was started and the platelet count promptly elevated; however, the liver function test did not respond as promptly as the platelet count and ursodeoxycholic acid was added, which led to an improvement of the liver function (Fig. 2). The platelet count was maintained with tapered doses of prednisolone and a percutaneous liver biopsy was performed six months after the start of prednisolone (Fig. 3). The biopsied specimen revealed an infiltration of chronic inflammatory cells with occasional plasma cells, interface hepatitis and focal necrosis in the small- and mid-sized portal area which was indicative of AIH. Lymphocytic infiltration to the bile ducts with degeneration of the bile ducts, intralobular granulomas, and copper deposition in the hepatocytes were also observed and considered to be characteristic of PBC. These histological findings supported the diagnosis of PBC-AIH overlap syndrome.

Currently, at 24 months since the full diagnosis was made, the patient's platelet count has remained steady at approximately $150 \times 10^{9} / \mathrm{L}$ and the liver enzymes are at the upper normal limits.

\section{Discussion}

It is well known that both $\mathrm{PBC}$ and $\mathrm{AIH}$ patients frequently have additional autoimmune diseases $(4,8)$. Antiplatelet autoantibodies against platelet glycoprotein (GP) IIb/ IIIa and GPIb/IX are detected in $40 \%$ of patients with $\operatorname{PBC}(5,6)$ and the cross-reactivity of the autoantibody to GPIIb/IIIa and michrochondrial M2 protein has been reported (16). Although a definite diagnosis of ITP is seldom made (4), it is assumed that the immune-mediated destruction of platelets plays a role in thrombocytopenia observed 


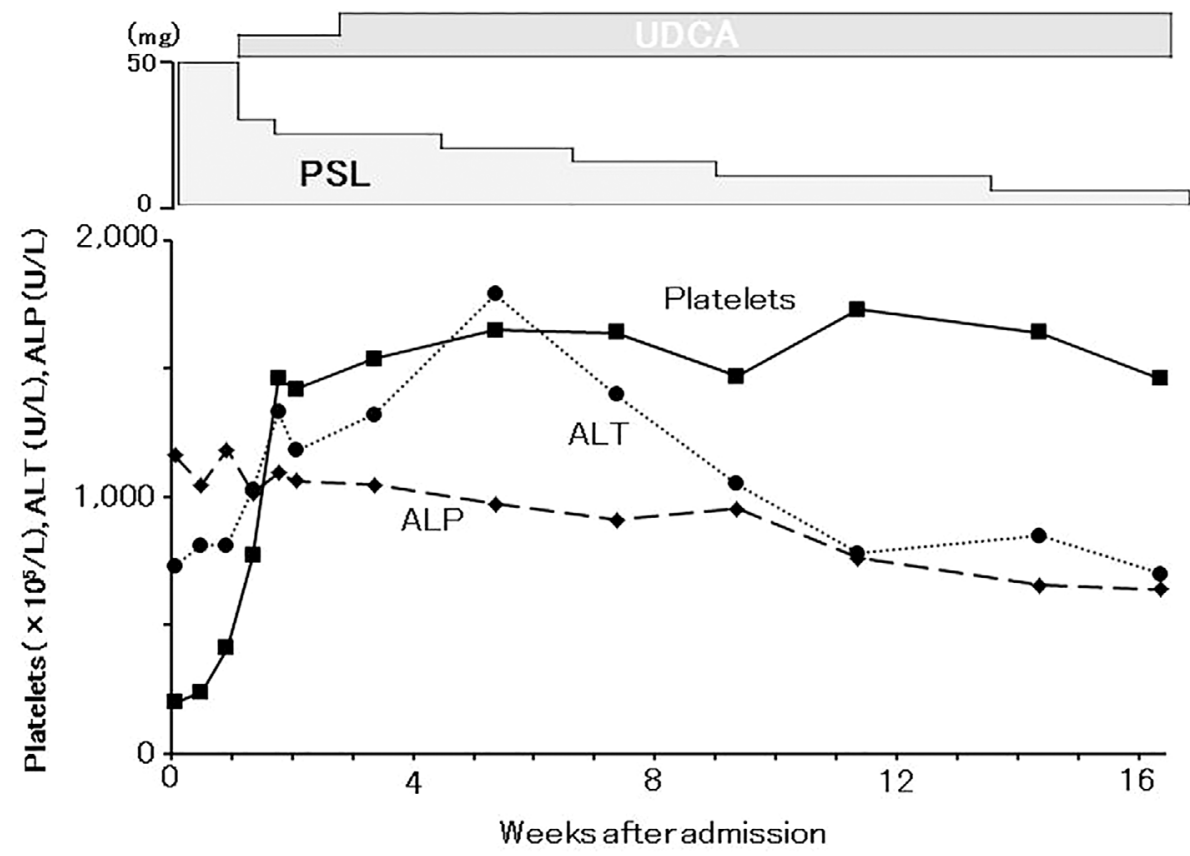

Figure 2. Changes in the platelet count, serum alanine aminotransferase (ALT), and alkaline phosphatase (ALP) following treatment. PSL: prednisolone, UDCA: ursodeoxycholic acid
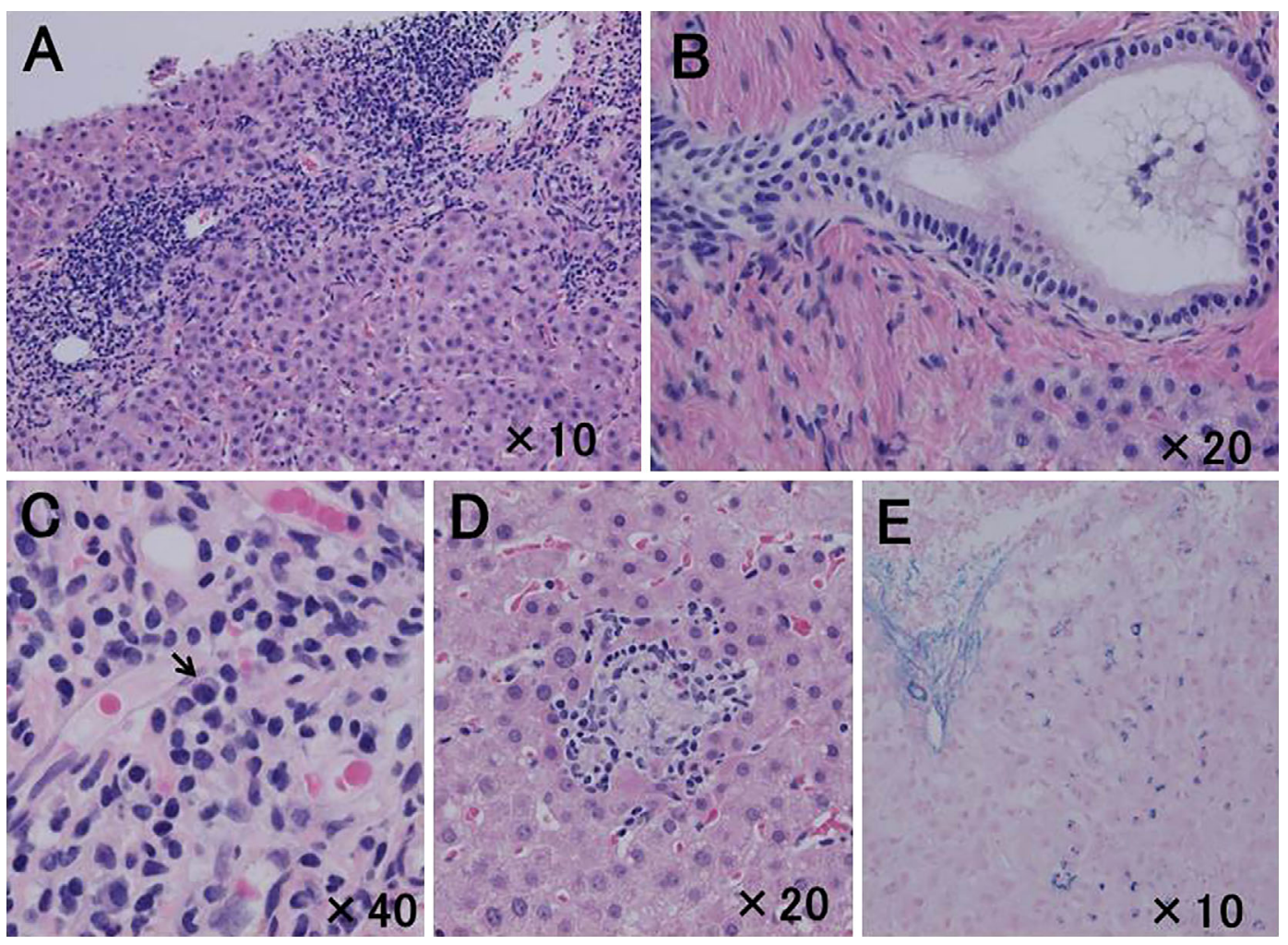

Figure 3. Histology of the liver biopsied six months after the start of steroid therapy. The liver biopsy demonstrates interface hepatitis $(\mathrm{A}, 10 \times)$ and the infiltration of chronic inflammatory cells with occasional plasma cells $(\mathrm{C}, 40 \times$, arrow), indicative of autoimmune hepatitis and the degeneration of the bile duct in the portal area $(B, 20 \times)$ and intralobular granulomas $(D, 20 \times)$ characteristic of primary biliary cirrhosis (PBC) (A-D, Hematoxylin and Eosin staining). Furthermore, copper deposition was observed in the hepatocytes (E, 10x, Victoria blue stain), which is also characteristic of PBC. 
in patients with $\operatorname{PBC}(5,6)$. On the contrary, the frequency of anti-platelet autoantibodies in patients with AIH has not yet been reported despite occasional case reports of AIH associated with ITP $(17,18)$. Similarly, while the frequency of extrahepatic autoimmune diseases among PBC-AIH overlap syndrome is reported to be as high as $43 \%$ (11), the frequency of anti-platelet autoantibodies has not yet been reported and there are currently only four reported cases of PBC-AIH overlap syndrome associated with ITP in the literature (12-15). The paucity of such association is not conceivable, considering the high prevalence of anti-platelet autoantibodies among patients with $\operatorname{PBC}(5,6)$. All four patients complicated with ITP had other autoimmune diseases which differed among the individual patients (12-15). One of them was complicated with Hashimoto's chronic thyroiditis (12); however, none had Grave's disease. Although the association between PBC-AIH overlap syndrome and immune-mediated thrombocytopenia is not widely recognized, it is speculated that immune-mediated mechanisms actually contribute to the development of thrombocytopenia in a considerable number of PBC-AIH patients. This association may be frequently overlooked as it is generally difficult to make a diagnosis of ITP in thrombocytopenic patients with chronic liver disease. In the present case, while non-immune-mediated mechanisms, including splenic destruction, should have contributed to thrombocytopenia to a certain extent, the rapid progression of thrombocytopenia, typical bone marrow findings, elevated serum PA IgG level, and prompt platelet response to steroid therapy suggested that immune-mediate mechanism was operative.

PBC-AIH overlap syndrome is a relatively newly defined entity (9) and its clinical characteristics have not been fully elucidated. Although the frequency of anti-platelet autoantibodies in this syndrome has not been reported, considering the similarly high frequencies of patients with $\mathrm{PBC}$ and PBC-AIH overlap syndrome who have extrahepatic autoimmune conditions $(4,11)$, it is assumed that the frequency of antiplatelet autoantibodies is similar to the frequency among patients with $\mathrm{PBC}$, which is approximately $40 \%(5,6)$. Moreover, the autoantibodies probably play a role in the mechanism of thrombocytopenia in a considerable portion of the patients with thrombocytopenia. As autoimmune thrombocytopenia in patients with PBC-AIH overlap syndrome may be responsive to steroid therapy, as exemplified by the present case, it is important to be aware of this medically treatable thrombocytopenia among patients with this syndrome. This case also illustrates the presence of global derangement of autoimmunity or shared genetic susceptibility in patients with PBC-AIH overlap syndrome.

The authors state that they have no Conflict of Interest (COI).

\section{References}

1. Marks PW. Hematologic manifestations of liver disease. Semin Hematol 50: 216-221, 2013.

2. Afdhal N, McHutchison J, Brown R, et al. Thrombocytopenia associated with chronic liver disease. J Hepatol 48: 1000-1007, 2008.

3. Jones DEJ. Autoantigens in primary biliary cirrhosis. J Clin Pathol 53: $813-821,2000$.

4. Watt FE, James OFW, Jones DEJ. Patterns of autoimmunity in primary biliary cirrhosis patients and their families: a populationbased cohort study. Q J Med 97: 397-406, 2004.

5. Bassendine MF, Collins JD, Stephenson J, Saunders P, James OFW. Platelet associated immunoglobulins in primary biliary cirrhosis: a cause of thrombocytopenia? Gut 26: 1074-1079, 1985.

6. Feistauer SM, Penner E, Mayr WR, Panzer S. Target platelet antigens of autoantibodies in patients with primary biliary cirrhosis. Hepatology 25: 1343-1345, 1997.

7. Hennes EM, Zeniya M, Czaja A, et al. Simplified criteria for the diagnosis of autoimmune hepatitis. Hepatology 48: 169-176, 2008.

8. Teufel A, Weinmann A, Kahaly GJ, et al. Concurrent autoimmune diseases in patients with autoimmune hepatitis. J Clin Gastroenterol 44: 208-213, 2010.

9. Chazouillères O, Wendum D, Serfaty L, Montembault S, Rosmorduc O, Poupon R. Primary biliary cirrhosis-autoimmune hepatitis overlap syndrome: clinical features and response to therapy. Hepatology 28: 296-301, 1998.

10. Neuhauser M, Bjornsson E, Treeprasertsuk S, et al. Autoimmune hepatitis-PBC overlap syndrome: A simplified scoring system may assist in the diagnosis. Am J Gastroenterol 105: 345-353, 2010.

11. Efe C, Wahlin S, Ozaslan E, et al. Autoimmune hepatitis/primary biliary cirrhosis overlap syndrome and associated extrahepatic autoimmune diseases. Eur J Gastroenterol Hepatol 24: 531-534, 2012.

12. Arakawa $Y$, Amaki S, Miyakawa H, et al. PBC-AIH overlap syndrome with concomitant ITP and Hashimoto's disease with positivity for anti-centromere antibody. J Gastroenterol 39: 490-495, 2004.

13. Toyoda M, Yokomori H, Kaneko F, et al. Primary biliary cirrhosis-autoimmune hepatitis overlap syndrome concomitant with systemic sclerosis, immune thrombocytopenic purpura. Intern Med 48: 2019-2023, 2009.

14. Kurihara Y, Shishido T, Oku K, et al. Polymyositis associated with autoimmune hepatitis, primary biliary cirrhosis, and autoimmune thrombocytopenic purpura. Mod Rheumatol 21: 325-329, 2011.

15. Korkmaz H, Bugdaci MS, Temel T, Dagli M, Karabagli P. Autoimmune hepatitis-primary biliary cirrhosis overlap syndrome concomitant with immune hemolytic anemia and immune thrombocytopenic purpura (Evans syndrome). Clin Res Hepatol Gastroenterol 37: e45-e50, 2013.

16. Panzer S, Penner E, Nelson PJ, Prochazka E, Benda H, Saurugger P. Identification of the platelet glycoprotein IIb/IIIa complex as a target antigen in primary biliary cirrhosis-associated autoimmune thrombocytopenia. J Autoimmunity 3: 473-483, 1990.

17. Santos ES, Arosemena LR, Raez LE, O'Brien C, Regev A. Successful treatment of autoimmune hepatitis and idiopathic thrombocytopenic purpura with the monoclonal antibody, rituximab: case report and review of literature. Liver Int 26: 625-629, 2006.

18. Yamaike N, Saigo K, Imoto $S$, et al. Autoimmune hepatitis accompanied by idiopathic thrombocytopenic purpura and Sjögren's syndrome. Intern Med 41: 72, 2002.

(C) 2015 The Japanese Society of Internal Medicine http://www.naika.or.jp/imonline/index.html 